\title{
Relação entre a Precipitação Anual e o Incremento em Volume de Árvores Amostradas em Plantações de Pinus taeda
}

\author{
Luiz Alberto Blanco Jorge ${ }^{1}$, Hideyo Aoki ${ }^{2}$, Ananias de Almeida Saraiva Pontinha ${ }^{3}$ \\ ${ }^{1}$ Departamento de Ciência Florestal, FCA - UNESP, Botucatu/SP, Brasil \\ ${ }^{2}$ Instituto Florestal do Estado de São Paulo, Floresta Estadual de Avaré, Avaré/SP, Brasil \\ ${ }^{3}$ Instituto Florestal do Estado de São Paulo, Estação Experimental de Itapeva, Itapeva/SP, Brasil
}

\begin{abstract}
RESUMO
O presente trabalho se baseou em análise de tronco completa para se verificar o comportamento do crescimento e do incremento corrente anual (ICA) em volume de quatro indivíduos de Pinus taeda amostrados em povoamentos localizados na Floresta Estadual de Avaré II e na Estação Experimental de Itapeva, Estado de São Paulo. Foram comparadas as séries temporais de precipitação e índice de ICA por meio dos coeficientes de correlação cruzada. Calcularam-se os coeficientes de convergência (GL) entre as séries. Para os indivíduos em que ocorreu significância estatística na correlação cruzada, a precipitação do ano anterior influiu significativamente no ICA em volume do ano considerado. Os valores de GL entre as séries foram maiores para Itapeva. Como não se observou diferença significativa nas distribuições das precipitações, credita-se para a textura do solo a 40-60 cm, média em Avaré e argilosa em Itapeva, o reflexo mais acentuado da disponibilidade de água sobre o ICA em volume na plantação de Itapeva.
\end{abstract}

Palavras-chave: análise de tronco completa, séries temporais, correlação cruzada.

\section{Relationship between Annual Rainfall and Timber Volume Increment of Trees Sampled in Pinus taeda plantations}

\begin{abstract}
This work was based on a complete tree stem analysis to assess the growth behavior and current annual volume increment (CAI) of four individuals of Pinus taeda sampled in populations located in the 'Avare II' State Forest and 'Itapeva' Experimental Station, State of São Paulo. The relationship between the rainfall time series and CAI index was examined by means of cross-correlation coefficients. The convergence coefficients (GL) between the series were calculated. In the individuals that showed a statistical significance in the cross-correlation between the time series, the previous year's rainfall significantly affected the current annual volume increment in the year under consideration. The GL values between the series were higher in 'Itapeva'. Since the two sites showed no significant difference in rainfall distribution, the difference was attributed to the soil texture at 40-60 cm - medium in 'Avare' and clayey in 'Itapeva', so the CAI of the 'Itapeva' plantation reflects the higher availability of water.
\end{abstract}

Keywords: complete stem analysis, time series, cross-correlation. 


\section{INTRODUÇÃO}

O perfil do tronco dos indivíduos de espécies arbóreas que emitem anéis de crescimento anuais pode ser reconstituído a partir da contagem e da medição dos anéis de seções transversais tomadas em diversas alturas. Por meio da análise de tronco completa, verificam-se os padrões do crescimento em camadas, o que permite o ajuste de modelos de funções de crescimento e incremento, conforme realizado por Barusso (1977) com árvores de Pinus elliottii e Pinus taeda amostradas em plantações florestais localizadas na região de Ponta Grossa, Estado do Paraná. As funções ajustadas descrevem o comportamento do crescimento passado relacionado a variáveis, como diâmetro, altura, fator de forma e volume. $\mathrm{O}$ entendimento do crescimento passado, registrado nos anéis de crescimento dos indivíduos, possibilita prognosticar o crescimento futuro. Para que se possa fazer uma prognose do crescimento relacionada aos povoamentos florestais e não somente aos indivíduos, deve-se proceder a uma amostragem intensiva, em que indivíduos de diferentes idades, sítios, classes fitossociológicas e classes de diâmetros estejam representados. De qualquer maneira, a medição dos anéis de crescimento se caracteriza por um trabalho lento e repetitivo. Buscando resolver esse problema, Rosot et al. (2003) desenvolveram um método digital para medição dos anéis, utilizando fotografia digital dos discos amostrados, processamento digital de imagens e sistema de informações geográficas.

Pinus taeda é uma espécie arbórea que possui, assim como a maioria das coníferas, uma distinção muito grande dos anéis de crescimento, ou seja, a cada ano é acrescentado um novo anel no tronco. Essa característica estimulou o desenvolvimento do projeto que se refletiu no artigo elaborado por Jorge et al. (2011) e se desdobrou na presente investigação. As duas pesquisas se complementam e constituem uma apreciação inicial do comportamento do crescimento passado da espécie em povoamentos localizados na Floresta Estadual de Avaré II e na Estação Experimental de Itapeva, unidades do Instituto Florestal do Estado de São Paulo. Os objetivos específicos da pesquisa foram: 1. A partir da análise de tronco completa, verificar o comportamento do crescimento e do incremento corrente anual em volume de indivíduos de Pinus taeda amostrados em povoamentos localizados nas duas unidades do Instituto Florestal citadas; 2. Analisar a relação entre a precipitação anual e o incremento corrente anual em volume das árvores.

\section{MATERIAL E MÉTODOS}

\section{1. Áreas de estudo}

A Floresta Estadual de Avaré II $\left(23^{\circ} 06^{\prime}\right.$ de latitude sul e $48^{\circ} 55^{\prime}$ de longitude oeste) e a Estação Experimental de Itapeva ( $24^{\circ} 02^{\prime}$ de latitude sul e $49^{\circ} 06^{\prime}$ de longitude oeste) se encontram em uma faixa de transição entre as regiões tropical e subtropical do Brasil, sendo a primeira unidade do Instituto Florestal do Estado de São Paulo ao norte e a segunda, ao sul do Trópico de Capricórnio: $23^{\circ}$ 26 ' 22" de latitude sul. O tipo climático da região segundo Köppen é o Cfa. A temperatura média do mês mais quente, que ocorre em janeiro, é de $22,3^{\circ} \mathrm{C}$ em Avaré e de $23,7^{\circ} \mathrm{C}$ em Itapeva. A temperatura média do mês mais frio é de $15,5^{\circ} \mathrm{C}$ (julho) em Avaré e de $17,3^{\circ} \mathrm{C}$ em Itapeva (junho). Informações acerca de precipitações médias mensais foram obtidas com base em dados de chuva de 1954 a 2008 para Avaré e de 1981 a 2008 para Itapeva (Figura 1). A precipitação média anual é igual a $1424 \mathrm{~mm} \mathrm{em}$ Avaré e $1378 \mathrm{~mm}$ em Itapeva. Quanto às unidades de solo, na Floresta Estadual de Avaré II, ocorre o predomínio de Latossolo Vermelho-Amarelo fase arenosa (LVa) e, na Estação Experimental de Itapeva, predomina Latossolo Vermelho Escuro-orto (LE). Nos dois talhões em que foram derrubados indivíduos de Pinus taeda com a finalidade de se realizar a análise de tronco, talhão 44 em Avaré e talhão 51 em Itapeva, também se efetuaram coletas de solo para se proceder a análise granulométrica (Tabela 1) e a análise química (Tabelas 2 e 3), de tal maneira a contribuir para uma caracterização geral dessas áreas de estudo. Para tanto, foram tomadas amostras compostas relacionadas às profundidades de $0-20 \mathrm{~cm}$ e $40-60 \mathrm{~cm}$.

\subsection{Seleção das árvores e de fatias retiradas do tronco}

O talhão 44 da Floresta Estadual de Avaré II, constituído por uma superfície de 3,70 ha, foi implantado em março de 1958 com uma densidade 
de 2500 indivíduos de Pinus taeda por hectare. Foram realizados três desbastes, aos 6, 16 e 26 anos. Em outubro de 2008, tendo a plantação 50 anos de idade, foram derrubados na área dois indivíduos para se proceder à análise de tronco completa. Inicialmente, foi feita uma varredura nas linhas de plantio, medindo-se os diâmetros à altura do peito (DAPs) de duzentas árvores, o que permitiu gerar

Precipitação média mensal

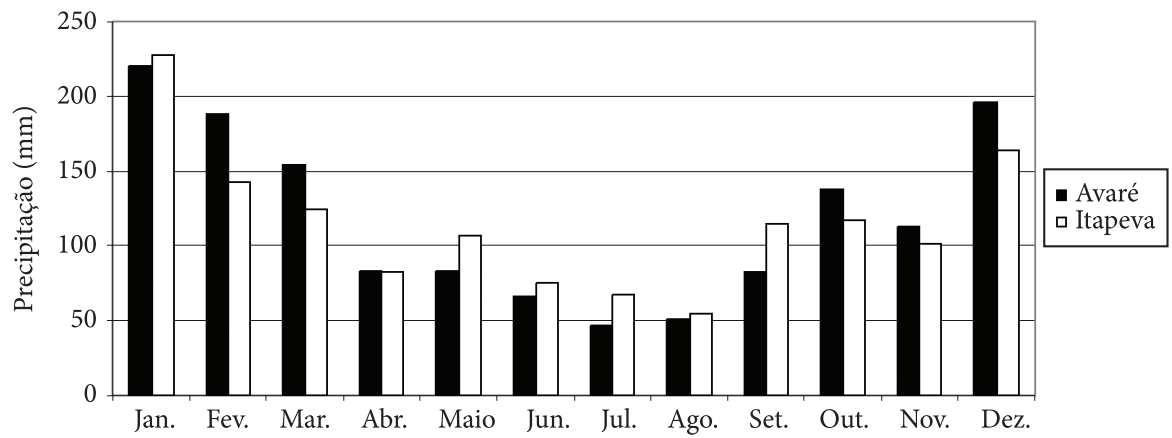

Figura 1. Distribuição de chuvas nas regiões de Avaré e Itapeva.

Figure 1. Rainfall distribution in the Avaré and Itapeva regions.

Tabela 1. Resultados da análise granulométrica do solo do talhão 44 em Avaré e do talhão 51 em Itapeva.

Table 1. Results of the granulometric analysis of the soil of stand 44 in Avaré and stand 51 in Itapeva.

\begin{tabular}{|c|c|c|c|c|c|c|c|}
\hline \multirow{2}{*}{\multicolumn{2}{|c|}{ Amostra }} & \multicolumn{3}{|c|}{ Areia } & \multirow{3}{*}{ Argila } & \multirow{3}{*}{ Silte } & \multirow{3}{*}{$\begin{array}{l}\text { Textura } \\
\text { do solo }\end{array}$} \\
\hline & & Grossa & Fina & Total & & & \\
\hline Talhão/local & Profundidade $(\mathrm{cm})$ & \multicolumn{3}{|r|}{$(\mathrm{g} / \mathrm{kg})$} & & & \\
\hline 44 Avaré & $0-20$ & 283 & 498 & 781 & 160 & 59 & Média \\
\hline 44 Avaré & $40-60$ & 251 & 512 & 763 & 179 & 58 & Média \\
\hline 51 Itapeva & $0-20$ & 304 & 249 & 552 & 345 & 103 & Média \\
\hline 51 Itapeva & $40-60$ & 316 & 241 & 557 & 353 & 90 & Argilosa \\
\hline
\end{tabular}

Tabela 2. Resultados da análise química/macronutrientes do solo do talhão 44 em Avaré e talhão 51 em Itapeva.

Table 2. Results of the macronutrient analysis of the soil of stand 44 in Avaré and stand 51 in Itapeva.

\begin{tabular}{|c|c|c|c|c|c|c|c|c|c|c|c|}
\hline \multicolumn{2}{|c|}{ Amostra } & pH & M.O. & $\mathbf{P}_{\text {resina }}$ & $\mathrm{H}+\mathrm{Al}$ & $\mathbf{K}$ & $\mathrm{Ca}$ & Mg & SB & CTC & V\% \\
\hline Talhão/local & Prof. (cm) & $\mathrm{CaCl}_{2}$ & $\mathrm{~g} / \mathrm{dm}^{3}$ & $\mathrm{mg} / \mathrm{dm}^{3}$ & \multicolumn{7}{|c|}{$\mathrm{mmol}_{\mathrm{c}} / \mathrm{dm}^{3}$} \\
\hline 44 Avaré & $0-20$ & 4,0 & 13 & 3 & 57 & 0,3 & 3 & 1 & 4 & 60 & 6 \\
\hline 44 Avaré & $40-60$ & 4,1 & 11 & 3 & 50 & 0,2 & 2 & 1 & 3 & 52 & 5 \\
\hline 51 Itapeva & $0-20$ & 6,3 & 8 & 28 & 11 & 1,6 & 25 & 5 & 31 & 43 & 74 \\
\hline 51 Itapeva & $40-60$ & 4,0 & 20 & 2 & 84 & 0,9 & 4 & 1 & 6 & 90 & 7 \\
\hline
\end{tabular}

Tabela 3. Resultados da análise química/micronutrientes do solo do talhão 44 em Avaré e talhão 51 em Itapeva. Table 3. Results of the micronutrient analysis of the soil of stand 44 in Avaré and stand 51 in Itapeva.

\begin{tabular}{|c|c|c|c|c|c|c|c|}
\hline \multicolumn{3}{|c|}{ Amostra } & Boro & Cobre & Ferro & Manganês & Zinco \\
\hline & o/local & Prof. (cm) & \multicolumn{5}{|c|}{$\mathrm{mg} / \mathrm{dm}^{3}$} \\
\hline 44 & Avaré & $0-20$ & 0,24 & 1,5 & 48 & 7,6 & 0,1 \\
\hline 44 & Avaré & $40-60$ & 0,23 & 1,7 & 38 & 4,3 & 0,1 \\
\hline 51 & Itapeva & $0-20$ & 0,16 & 1,3 & 16 & 20,3 & 3,0 \\
\hline 51 & Itapeva & $40-60$ & 0,26 & 1,1 & 75 & 1,5 & 0,1 \\
\hline
\end{tabular}


a distribuição de diâmetros, como representado na Figura 2a. Selecionaram-se os dois indivíduos em função da distribuição nas classes de tamanho. O primeiro, com dimensões de $32,0 \mathrm{~cm}$ de DAP e $27,0 \mathrm{~m}$ de altura total, referente à classe de diâmetro médio $(29,0 \mid-32,5 \mathrm{~cm})$, e o segundo, com dimensões de $42 \mathrm{~cm}$ de DAP e 28,7 $\mathrm{m}$ de altura total, sendo uma árvore codominante (classe de diâmetro: $39,5 \mid-43,0 \mathrm{~cm})$.

O talhão 51 da Estação Experimental de Itapeva, constituído por uma superfície de 19,19 ha, foi implantado em 1981 com uma densidade de 1667 indivíduos de Pinus taeda por hectare. Foram realizados quatro desbastes, aos 9, 14, 20 e 27 anos. Em outubro de 2009, tendo a plantação 28 anos de idade, foram derrubados na área dois indivíduos para se proceder à análise de tronco completa. Inicialmente, foi feita uma varredura nas linhas de plantio, medindo-se os diâmetros à altura do peito (DAPs) de duzentas árvores, o que permitiu gerar a distribuição de diâmetros, como representado na Figura 2b. Selecionaram-se os dois indivíduos em função da distribuição nas classes de tamanho. O primeiro, com dimensões de $31,9 \mathrm{~cm}$ de DAP e $25,6 \mathrm{~m}$ de altura total, referente à classe de diâmetro médio $(29,0 \mid-32,5 \mathrm{~cm})$, e o segundo, com dimensões de 39,9 cm de DAP e 24,5 m de altura total, sendo uma árvore codominante (classe de diâmetro: $39,5 \mid-43,0 \mathrm{~cm})$.
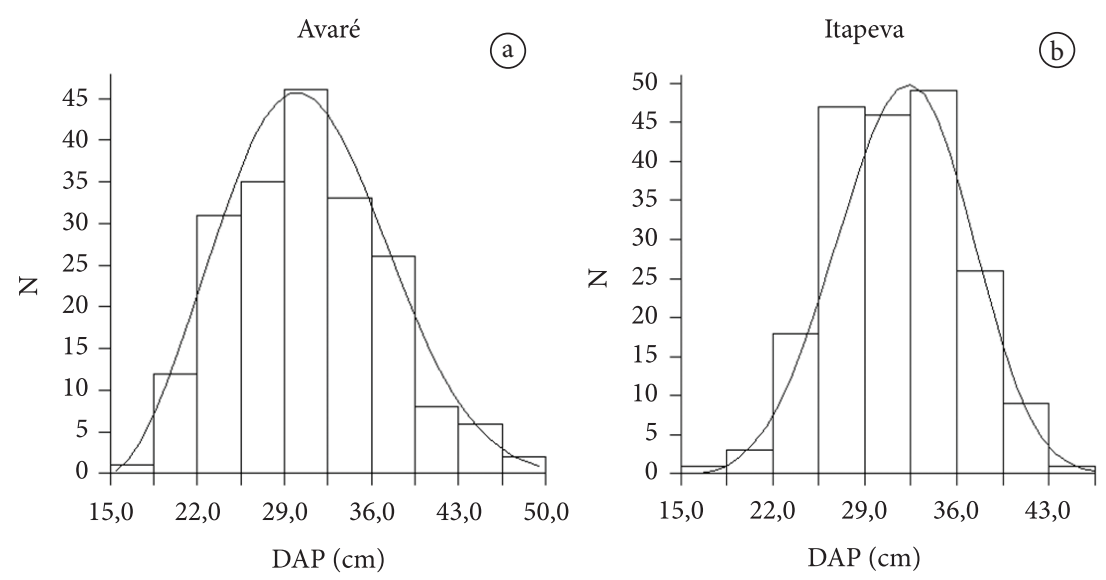

Figura 2. Distribuições diamétricas dos povoamentos de Pinus taeda: a) talhão 44 da Floresta Estadual de Avaré II, com 50 anos de idade; b) talhão 51 da Estação Experimental de Itapeva, com 28 anos de idade.

Figure 2. Diameter distributions of the communities of Pinus taeda: a) stand 44 of the 50-year-old Avaré II State-owned Forest; b) stand 51 of the 28-year-old Itapeva Experimental Farm. 
imediatamente anterior (Barusso, 1977; Rosot et al., 2003).

A verificação do comportamento do crescimento e do incremento corrente anual dos indivíduos amostrados demandou os cálculos de volumes relacionados aos anéis de crescimento (Jorge et al., 2011). As alturas de término de anel e os volumes observados dascamadas decrescimento foramobtidos após o desenvolvimento, no ambiente do Microsoft Access, de módulos de programação com o Visual Basic for Applications - VBA. O comportamento do crescimento em volume dos indivíduos foi descrito por meio de equações ajustadas a partir de um modelo modificado (Equação 1) da função de incremento de Hugershoff (Prodan, 1968):

$v=a t^{m} e^{k t^{\gamma}}$

em que: $\mathrm{v}=$ volume acumulado até a camada de crescimento $\left(\mathrm{m}^{3}\right) ; \mathrm{t}=$ idade em anos; $\mathrm{a}, \mathrm{m}$, $\mathrm{k}=$ coeficientes do modelo de equação; $\gamma=$ potência.

Tendo-se como base o modelo linearizado (Equação 2), usaram-se as equações lineares como funções de autoiniciação para o ajuste das equações não lineares no ambiente do software estatístico R (Ritz \& Streibig, 2008), sendo os valores iniciais dos coeficientes de a, $\mathrm{m}, \mathrm{k}$ assumidos, respectivamente, por $\mathrm{e}^{\mathrm{b} 0}, \mathrm{~b}_{1}, \mathrm{~b}_{2}$.

$\ln v=b_{0}+b_{1} \ln t+b_{2} t^{\gamma}$

\subsection{Relação entre a precipitação anual e o incremento em volume das árvores}

Takahashi et al. (2005), Wilczynski \& Feliksik (2007) e Feliksik \& Wilczynski (2008) estudaram a influência das condições climáticas sobre a largura dos anéis de crescimento, usando para tanto variáveis, como temperatura e precipitação. Em face da disponibilidade de dados históricos apenas de precipitação, no presente trabalho, avaliou-se a relação entre a precipitação acumulada durante um ano e o índice de incremento corrente anual (ICA) em volume dos troncos das árvores amostradas. $\mathrm{O}$ índice de ICA (cronologia residual) foi calculado, de acordo com Fritts (1976), usando-se a Equação 3:

$I_{i}=R_{i} \cdot Y_{i}^{-1}$

em que: $\mathrm{I}=$ índice de ICA em volume; $\mathrm{R}=\mathrm{ICA}$ em volume do anel de crescimento; $\mathrm{Y}=$ valor da curva ajustada; $\mathrm{i}=$ ano.

O modelo utilizado para ajustar a curva, cujo valor se refere ao denominador da fórmula do índice de ICA em volume, foi um polinômio de nono grau sem o $b_{0}$, isto é, saindo da origem, em que o ICA em volume foi dado como uma função da idade.

Analisou-se a relação da precipitação anual com o índice de incremento corrente anual em volume das árvores, por meio dos coeficientes de correlação cruzada (Ehlers, 2009) calculados a partir dos dados das respectivas séries temporais. Empregou-se também um método de similaridade baseado no coeficiente de convergência - GL (Feliksik \& Wilczynski, 2003), calculado pela Equação 4:

$G L=100 \cdot m \cdot(n-1)^{-1}$

em que: $\mathrm{m}=$ número de seções convergentes das curvas comparadas; $\mathrm{n}=$ número de anos comparados.

\section{RESULTADOS E DISCUSSÃO}

Foi aplicado, no ambiente do software estatístico $\mathrm{R}$, o teste de Shapiro-Wilk aos dados de precipitações anuais de Avaré e Itapeva, relativos aos períodos de tempo analisados. Verificou-se que as distribuições dos dados (Figura 3) apresentaram normalidade (Tabela 4). As séries temporais obtidas (Figura 4)

Tabela 4. Estatísticas relacionadas ao teste de Shapiro-Wilk para normalidade.

Table 4. Statistics of the Shapiro-Wilk normality test.

\begin{tabular}{lcc}
\hline \multicolumn{1}{c}{ Variável } & Estatística W & p-valor \\
\hline Precipitação anual - Avaré & 0,9641 & 0,1479 \\
\hline Índice de ICA - árvore de diâmetro médio/Avaré & 0,9832 & 0,7176 \\
\hline Índice de ICA - árvore codominante/Avaré & 0,9670 & 0,1935 \\
\hline Precipitação anual - Itapeva & 0,9476 & 0,2214 \\
Índice de ICA - árvore de diâmetro médio/Itapeva & 0,9664 & 0,5558 \\
Índice de ICA - árvore codominante/Itapeva & 0,9746 & 0,7618 \\
\hline
\end{tabular}



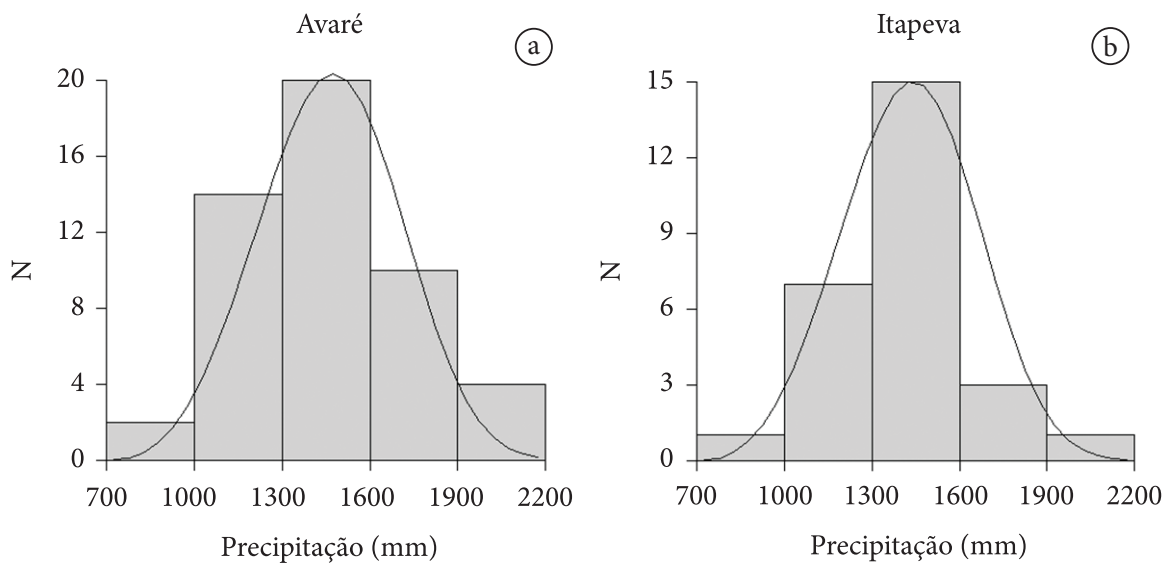

Figura 3. Distribuições das precipitações anuais: a) Avaré; b) Itapeva.

Figure 3. Annual rainfall distribution: a) Avaré; b) Itapeva.
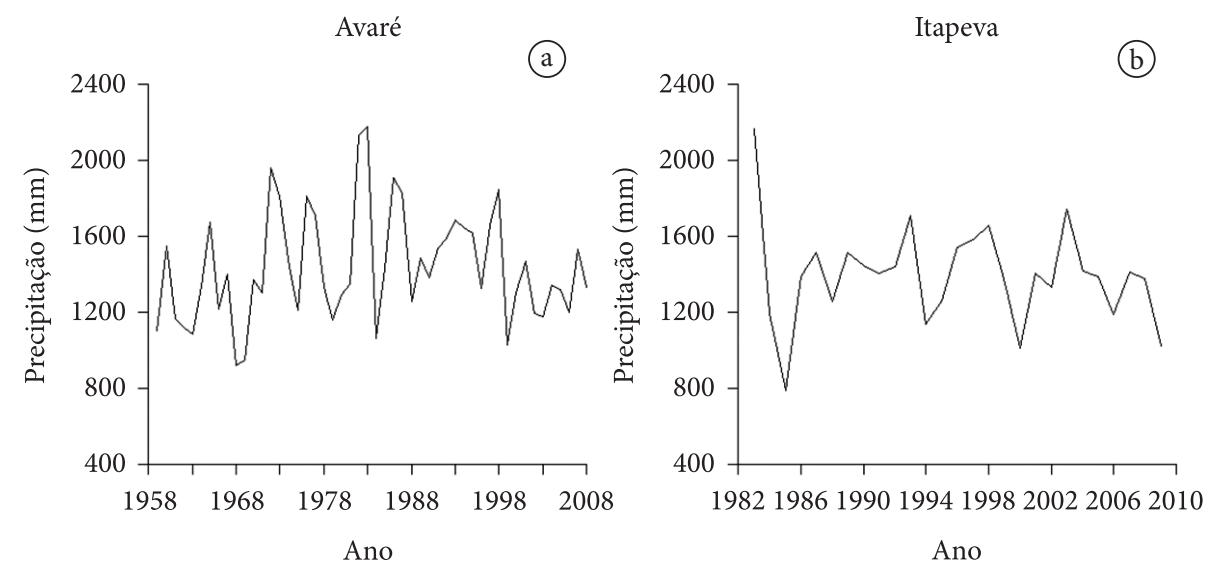

Figura 4. Séries temporais de precipitação anual (mm): a) Avaré; b) Itapeva.

Figure 4. Time series of annual rainfall ( $\mathrm{mm})$ : a) Avaré; b) Itapeva.

puderem ser comparadas com aquelas de índice de ICA das árvores amostradas.

Tendo sido gerados os perfis dos troncos - como o observado para a árvore de diâmetro médio amostrada em Avaré (Figura 5) -, calculados os volumes das camadas e ajustada a equação de crescimento para cada árvore (Tabela 5; Figuras 6a-d), pôde-se verificar o comportamento do crescimento em volume dos indivíduos de Pinus taeda selecionados em Avaré e Itapeva.

A razão entre os valores observados e estimados do ICA em volume das árvores (Figura 7) constituiu as séries do índice de ICA - cronologia residual (Figura8), necessáriasparaa comparação com as séries de precipitação anual dos locais correspondentes. Os valores do índice de ICA dos dois primeiros anos das quatro árvores amostradas foram considerados como pontos aberrantes (outliers), sendo retirados da análise comparativa entre séries temporais. Após essa retirada, os dados de índice de ICA em volume das árvores apresentaram normalidade pelo teste de Shapiro-Wilk (Tabela 4). Os valores de correlação cruzada, gerados no ambiente do software estatístico $\mathrm{R}$, entre a precipitação anual e o índice de ICA em volume dos indivíduos de Pinus taeda (Figura 9) - observando-se os limites de confiança de 95 \% (Ehlers, 2009) e considerando-se a defasagem de um ano - apresentaram valores com significância 
Pinus taeda - Avaré

DAP: $32 \mathrm{~cm}$

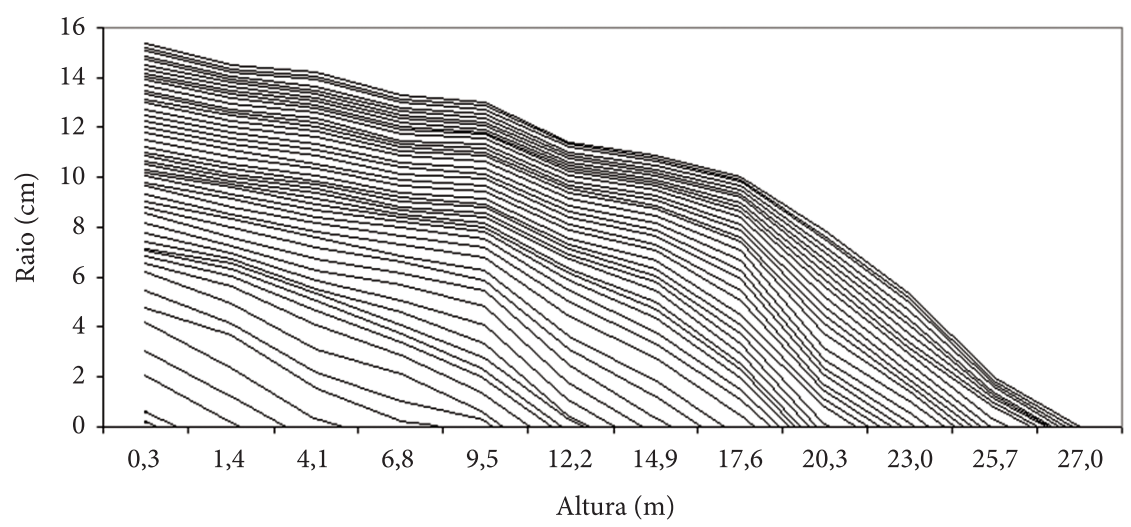

Figura 5. Perfil do tronco de indivíduo de Pinus taeda com $32 \mathrm{~cm}$ de DAP e 50 anos de idade, Avaré/SP.

Figure 5. Tree trunk profile of a 50-year-old individual of Pinus taeda with a DBH of $32 \mathrm{~cm}$, Avaré, SP.

(a)

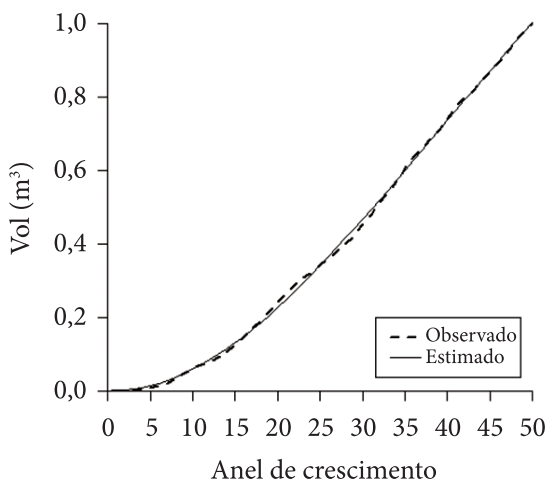

(c)

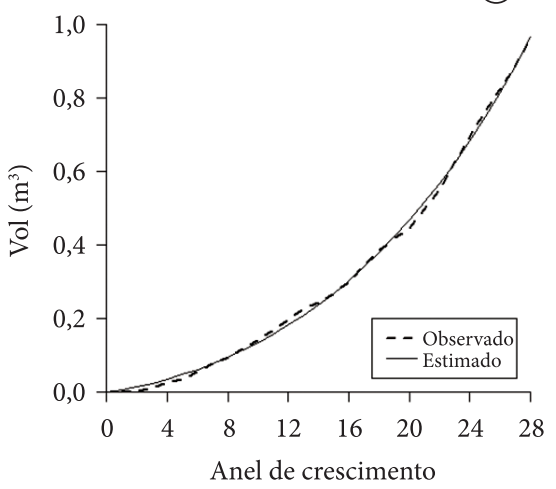

(b)

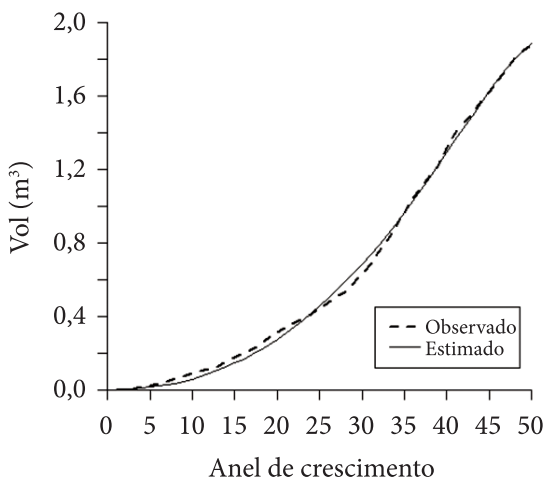

(d)

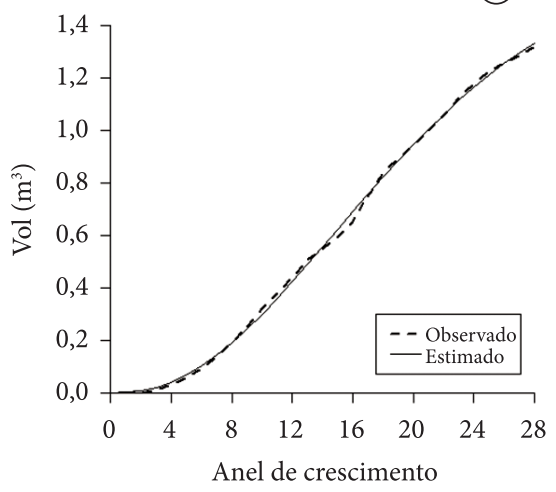

Figura 6. Crescimento em volume de indivíduos de Pinus taeda: a) árvore com $32 \mathrm{~cm}$ de DAP e 50 anos de idade, Avaré-SP; b) árvore com $42 \mathrm{~cm}$ de DAP e 50 anos de idade, Avaré-SP; c) árvore com 31,9 cm de DAP e 28 anos de idade, Itapeva-SP; d) árvore com 39,9 cm de DAP e 28 anos de idade, Itapeva-SP.

Figure 6. Volume growth of Pinus taeda individuals: a) 50-year-old tree with a DBH of $32 \mathrm{~cm}$, Avaré, SP; b) 50-yearold tree with a DBH of $42 \mathrm{~cm}$, Avaré, SP; c) 28 -year-old tree with a DBH of $31.9 \mathrm{~cm}$, Itapeva, SP; d) 28 -year-old tree with a $\mathrm{DBH}$ of $39.9 \mathrm{~cm}$, Itapeva, SP. 
Tabela 5. Estatísticas relacionadas às equações de crescimento ajustadas.

Table 5. Statistics of the adjusted growth functions.

\begin{tabular}{lcccccccc} 
Local & $\mathbf{D A P}(\mathbf{c m})$ & $\mathbf{a}$ & $\mathbf{m}$ & $\mathbf{k}$ & $\gamma$ & $\mathbf{s y x}$ & $\mathbf{s y x} \%$ & $\mathbf{n}^{\mathbf{0}}$ iterações \\
\hline Avaré & 32,0 & $0,00038764^{*}$ & $2,25658038^{*}$ & $-0,01937158^{*}$ & 1 & 0,008439 & 2,09 & 31 \\
\hline Avaré & 42,0 & $0,00032336^{*}$ & $2,25145500^{*}$ & $-1,38699 \mathrm{e}-18^{*}$ & 10 & 0,026650 & 3,99 & 6 \\
\hline Itapeva & 31,9 & $0,00502522^{*}$ & $1,25887773^{*}$ & $0,03800941^{*}$ & 1 & 0,010670 & 3,20 & 23 \\
\hline Itapeva & 39,9 & $0,00132527^{*}$ & $2,65472281^{*}$ & $-0,06906692^{*}$ & 1 & 0,014600 & 2,39 & 27 \\
\hline
\end{tabular}

${ }^{*} \mathrm{P} \leq 0,001$.
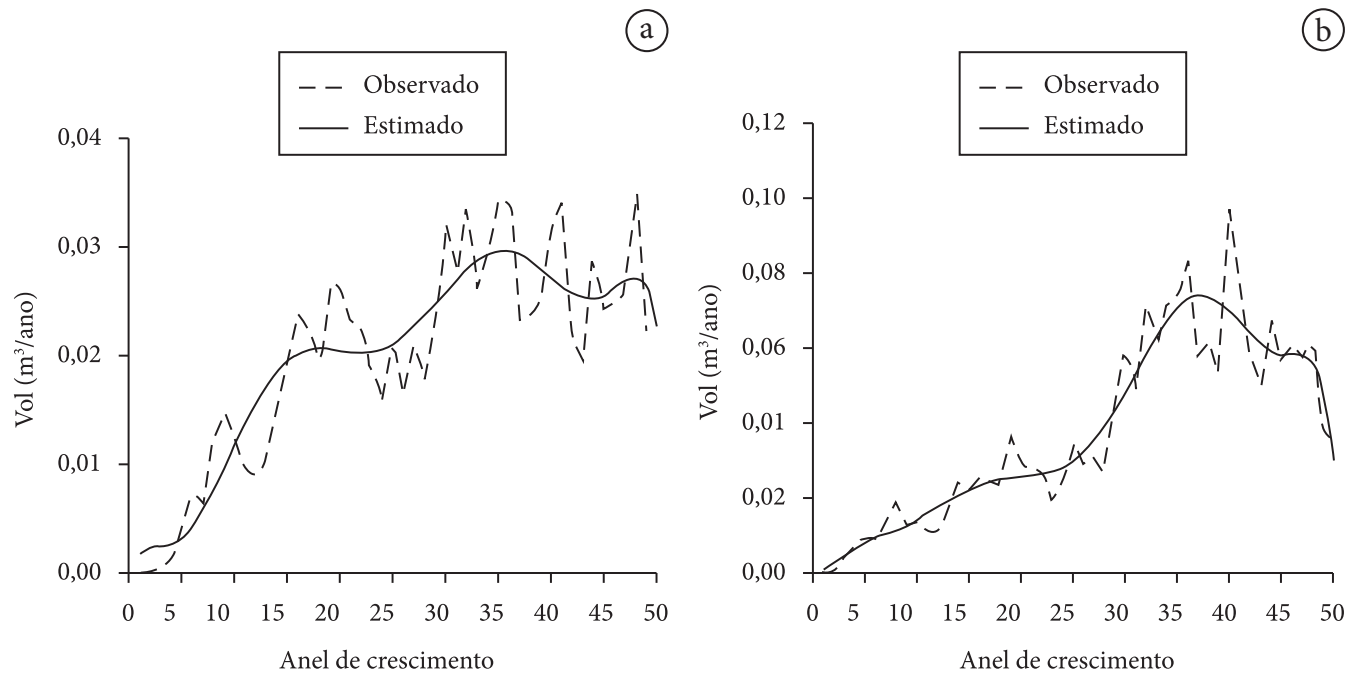

(c)
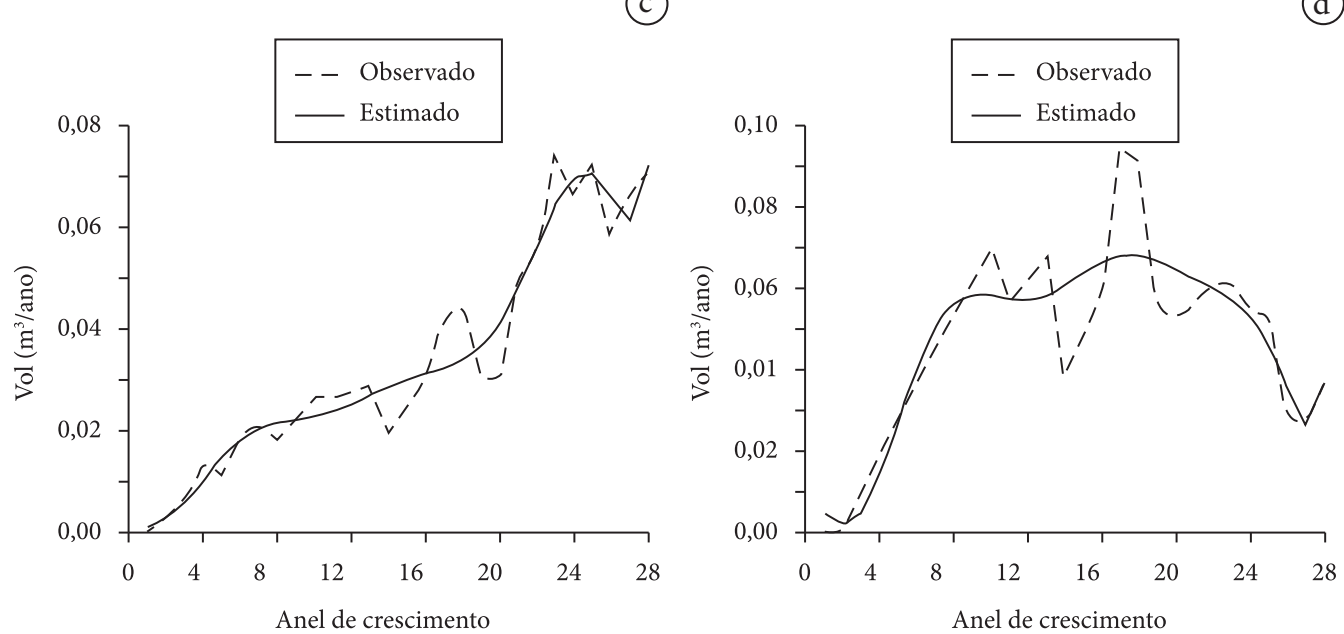

Figura 7. Valores observados e estimados de incremento corrente anual (ICA) em volume de indivíduos de Pinus taeda: a) árvore com $32 \mathrm{~cm}$ de DAP e 50 anos de idade, Avaré-SP; b) árvore com $42 \mathrm{~cm}$ de DAP e 50 anos de idade, Avaré-SP; c) árvore com 31,9 cm de DAP e 28 anos de idade, Itapeva-SP; d) árvore com 39,9 cm de DAP e 28 anos de idade, Itapeva-SP.

Figure 7. Observed and estimated CAI of Pinus taeda individuals: a) 50-year-old tree with $32 \mathrm{~cm}$ of DBH, Avaré/SP; b) 50-year-old tree with $42 \mathrm{~cm}$ of DBH, Avaré/SP; c) 28 -year-old tree with $31.9 \mathrm{~cm}$ of DBH, Itapeva/SP; d) 28-yearold tree with $39.9 \mathrm{~cm}$ of DBH, Itapeva/SP. 
estatística para os indivíduos codominantes de Avaré e Itapeva, e para a árvore de diâmetro médio de Itapeva. Não houve significância na correlação cruzada para a árvore de diâmetro médio de Avaré. Complementou-se a avaliação calculando-se os valores de coeficiente de convergência (GL) entre as duas séries, levando-se em conta a defasagem de um ano. Esses valores de GL foram de 57,45\%, $63,83 \%, 83,33 \%$ e $75,00 \%$, respectivamente, para os indivíduos de diâmetro médio e codominante de Avaré, e de diâmetro médio e codominante de Itapeva.
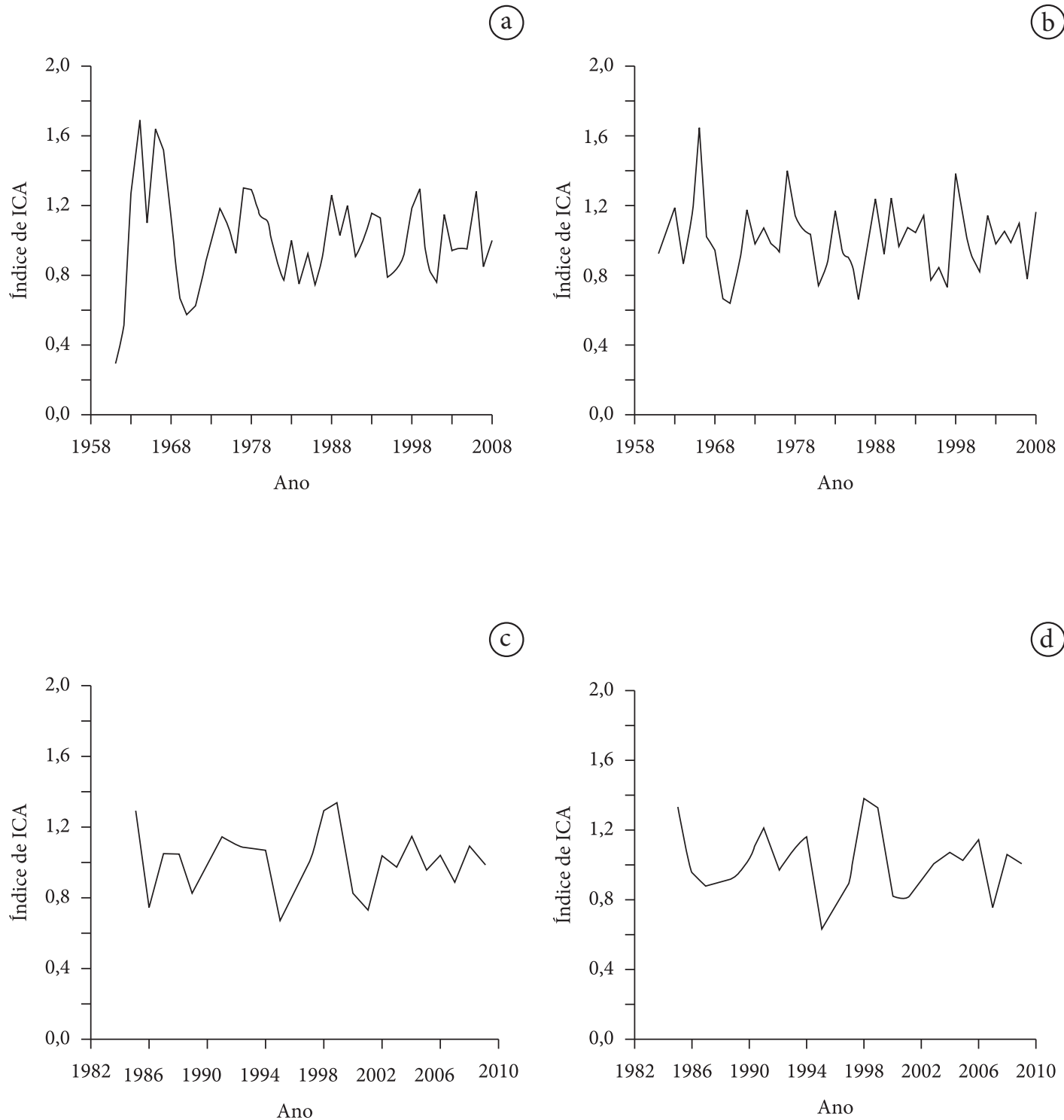

Figura 8. Séries temporais do índice de ICA em volume de indivíduos de Pinus taeda: a) árvore com $32 \mathrm{~cm}$ de DAP e 50 anos de idade, Avaré-SP; b) árvore com $42 \mathrm{~cm}$ de DAP e 50 anos de idade, Avaré-SP; c) árvore com 31,9 cm de DAP e 28 anos de idade, Itapeva-SP; d) árvore com 39,9 cm de DAP e 28 anos de idade, Itapeva-SP.

Figure 8. Time series of the CAI index of Pinus taeda individuals: a) 50-year-old tree with $32 \mathrm{~cm}$ of DBH, Avaré/SP; b) 50 -year-old tree with $42 \mathrm{~cm}$ of DBH, Avaré/SP; c) 28 -year-old tree with $31.9 \mathrm{~cm}$ of DBH, Itapeva/SP; d) 28-yearold tree with $39.9 \mathrm{~cm}$ of DBH, Itapeva/SP. 
(a)
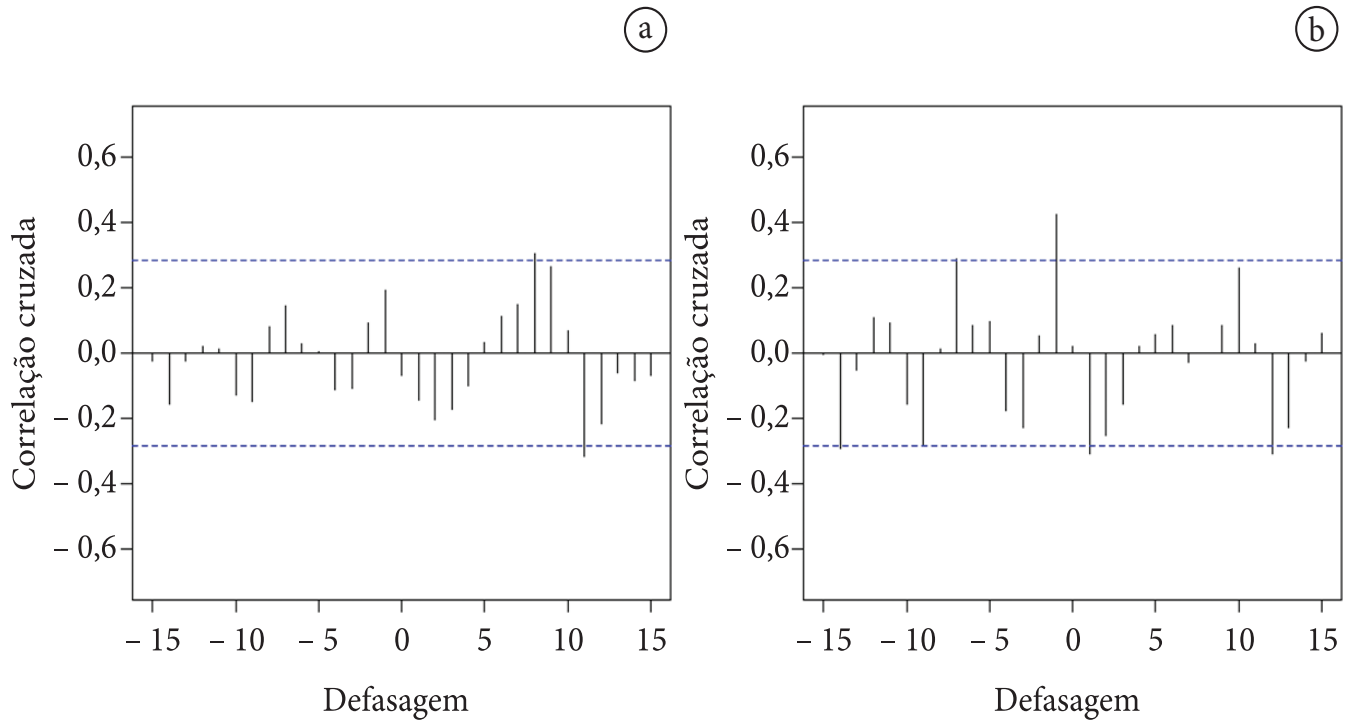

(c)
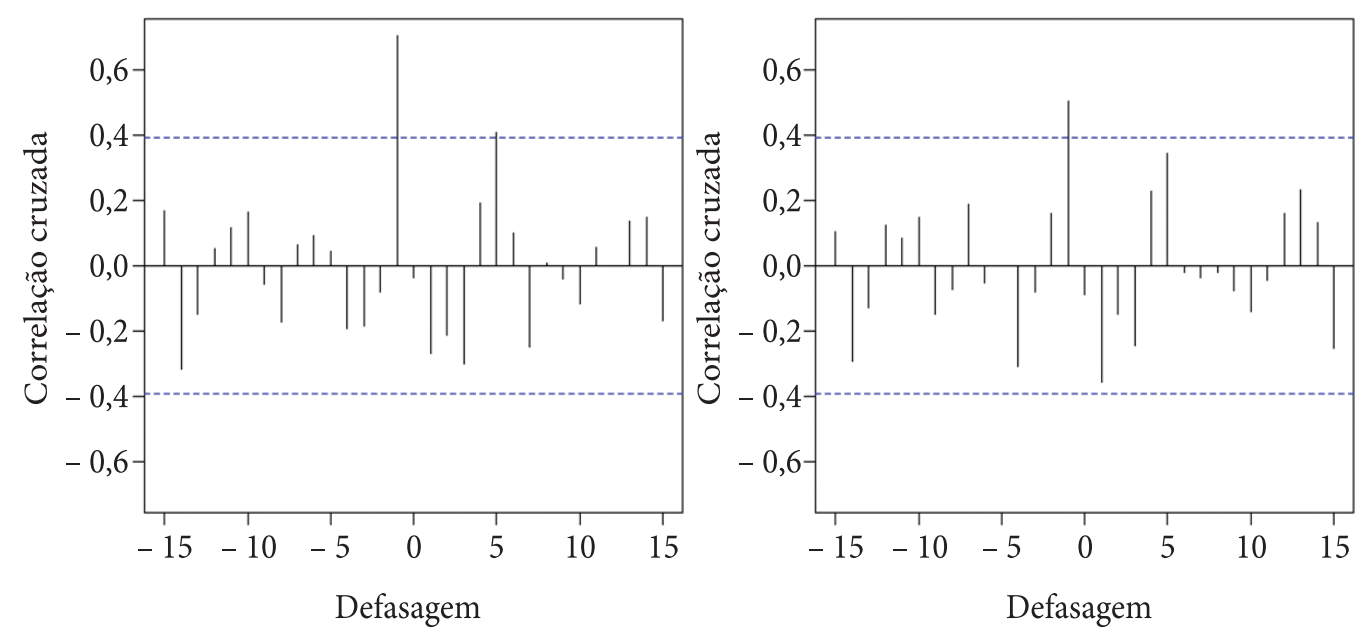

Figura 9. Correlação cruzada entre a precipitação anual e o índice de ICA em volume de indivíduos de Pinus taeda: a) árvore com $32 \mathrm{~cm}$ de DAP e 50 anos de idade, Avaré-SP; b) árvore com $42 \mathrm{~cm}$ de DAP e 50 anos de idade, AvaréSP; c) árvore com 31,9 cm de DAP e 28 anos de idade, Itapeva-SP; d) árvore com 39,9 cm de DAP e 28 anos de idade, Itapeva-SP.

Figure 9. Cross-correlation between annual rainfall and the CAI index of Pinus taeda individuals: a) 50-year-old tree with $32 \mathrm{~cm}$ of DBH, Avaré/SP; b) 50-year-old tree with $42 \mathrm{~cm}$ of DBH, Avaré/SP; c) 28-year-old tree with $31.9 \mathrm{~cm}$ of DBH, Itapeva/SP; d) 28-year-old tree with $39.9 \mathrm{~cm}$ of DBH, Itapeva/SP.

\section{CONCLUSÕES E RECOMENDAÇÃO}

Com base nos resultados do presente trabalho de pesquisa, concluiu-se que:

- A pequena modificação implementada na função de incremento de Hugershoff permitiu usar o modelo para ajustar adequadamente as equações de crescimento em volume das árvores, como observado pelo nível de significância dos estimadores dos coeficientes e pelos erros padrões de estimativa encontrados;

- Para os indivíduos amostrados em que ocorreu significância estatística na correlação cruzada entre as séries temporais de precipitação anual e índice de ICA em volume, houve um indicativo de que a precipitação do ano anterior influiu 
significativamente no incremento corrente anual em volume do ano considerado; e

- Os valores dos coeficientes de convergência entre as séries temporais foram maiores para Itapeva. Entretanto, como não ocorreu diferença significativa nas distribuições das precipitações entre os dois locais, credita-se para a textura do solo à profundidade de 40-60 cm, média em Avaré e argilosa em Itapeva, o reflexo mais acentuado da disponibilidade de água sobre o incremento corrente anual em volume na plantação de Itapeva.

Recomenda-se a continuação dos estudos de crescimento com suporte em análise de tronco. Sugere-se o desdobramento em um projeto em que se intensifique a amostragem realizada nas plantações, considerando-se primeiro uma classificação de sítios e, em seguida, a seleção de indivíduos que leve em conta, de forma hierárquica, a idade, o sítio e as classes de diâmetro dos povoamentos florestais.

\section{AGRADECIMENTOS}

Gostaríamos de agradecer ao Instituto Florestal do Estado de São Paulo por ceder o material que permitiu o desenvolvimento do presente trabalho.

Esta pesquisa é dedicada a Altair Pereira Barusso, Engenheiro Florestal, aluno do Curso de PósGraduação em Ciências Florestais da Universidade Federal do Paraná, que deixou o plano físico pouco tempo antes de poder defender sua Dissertação de Mestrado. O Trabalho de Dissertação do Altair é fonte de inspiração para todos aqueles que buscam se aprofundar nos estudos de análise de tronco e crescimento florestal.

\section{STATUS DA SUBMISSÃO}

Recebido: 07/05/2012

Aceito: $10 / 07 / 2012$

Publicado: 31/12/2012

\section{AUTOR(ES) PARA CORRESPONDÊNCIA}

\section{Luiz Alberto Blanco Jorge}

Departamento de Ciência Florestal - DRN, Faculdade de Ciências Agronômicas - FCA, Universidade Estadual Paulista - UNESP, Rua Doutor José Barbosa de Barros, 1780, Fazenda Experimental Lageado, CEP 18610-307, Botucatu, SP, Brasil e-mail: blanco@fca.unesp.br

\section{REFERÊNCIAS}

Barusso AP. Determinação de funções de crescimento mediante análise de tronco [dissertação]. Curitiba: Setor de Ciências Agrárias, Universidade Federal do Paraná; 1977. Não defendida.

Ehlers RS. Análise de séries temporais. 5th ed. São Carlos: Ricardo Sandes Ehlers; 2009. 114 p.

Feliksik E, Wilczynski S. Diversification of increment reactions of Douglas fir (Pseudotsuga menziesii Franco) from mountainous regions of southern Poland. Journal of Forest Science 2003; 49(12): 552-558.

Feliksik E, Wilczynski S. Tree-ring chronology as a source of information on susceptibility of Sitka Spruce to climatic conditions of Pomerania (Northern Poland). Geochronometria 2008; 30: 79-82. http://dx.doi. org/10.2478/v10003-008-0002-0

Fritts HC. Tree-rings and climate. New York: Academic Press; 1976. 567 p.

Jorge LAB, Aoki H, Pontinha AAS. Análise de tronco completa de árvores amostradas em plantações de Pinus taeda localizadas em Avaré e Itapeva, Estado de São Paulo. In: Anais do V Simpósio Latino-Americano sobre Manejo Florestal; 2011; Santa Maria. Santa Maria: Universidade Federal de Santa Maria; 2011. p. 59-69.

Prodan M. Forest biometrics. London: Pergamon Press; 1968. 447 p.

Ritz C, Streibig JC. Nonlinear regression with $R$. New York: Springer; 2008. 144 p.

Rosot MAD, Figueiredo Filho A, Disperati AA, Emerenciano DB. Análise de tronco digital: uma nova medição de anéis de crescimento. Revista Floresta 2003; 33(3): 235-255.

Takahashi K, Tokumitsu Y, Yasue K. Climatic factors affecting the tree-ring widht of Betula ermanii at the timberline on Mount Norikura, Central Japan. Ecological Research 2005; 20: 445-451. http://dx.doi. org/10.1007/s11284-005-0060-y

Wilczynski S, Feliksik E. Local chronologies and regional diversity of dendrochronological signal of Douglas Fir in Poland. Geochronometria 2007; 26: 69-80. http:// dx.doi.org/10.2478/v10003-007-0008-z 